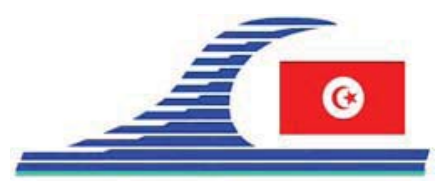

Conférence Méditerranéenne Côtière et Maritime

EDITION 1, HAMMAMET, TUNISIE (2009)

Coastal and Maritime Mediterranean Conference

Disponible en ligne - http://www.paralia.fr-Available online

\title{
Comportement mécanique de la tangue traitée avec un liant et un produit chimique réactif
}

\author{
Makiko OBANA ${ }^{1}$, Daniel LEVACHER ${ }^{2}$, Philippe DHERVILLY ${ }^{3}$
}

1. Université de Nagoya, Nagoya, Japon.

mobana@civil.nagoya-u.ac.jp

2. Université de Caen, Faculté des Sciences, UMR 6143 CNRS - M2C,

Esplanade de la Paix, 14032 Caen, France.

daniel.levacher@unicaen.fr

3. ARD Normandie, 14000 Hérouville Saint-Clair, France.

pdhervilly@ard-normandie.fr

\section{Résumé :}

Le projet de réaménagement de la baie du Mont-Saint-Michel prévoit le déblaiement de plus d'un million de $\mathrm{m}^{3}$ de sédiments. De nombreuses interrogations se posent sur leur devenir. En effet les seules filières de valorisation locales concernent l'amendement agricole et ne peuvent éliminer de telles quantités. Pour assurer la gestion à terre et l'élimination de ces sédiments non contaminés, la recherche de nouvelles voies de valorisation s'est imposée. Une des filières potentielles envisagée est celle de l'élaboration d'un matériau pour sous couches routières ou voies piétonnes, et en particulier pour un réemploi local au niveau du projet. L'étude proposée a concerné la valorisation de ces sédiments, appelés communément tangues, à l'aide d'un traitement par liant hydraulique et par alcali activation. Des essais de faisabilité de stabilisation/solidification sur la tangue ont porté sur l'étude de mélanges d'une part, de tangue et de liant pouzzolanique, et d'autre part, de tangue et d'un produit d'alcali activation. Un traitement de surface a été appliqué sur une série d'éprouvettes de ces deux types de mélanges à l'aide d'un produit de cure. L'analyse comparée des deux procédés de stabilisation s'est faite sur la base des résultats d'essais réalisés sur les mélanges: résistance mécanique et durabilité. Les possibilités d'emploi d'un tel traitement sont discutées en tenant compte des recommandations en vigueur.

\section{Mots-clés :}

Sédiment marin - Tangue - Stabilisation - Liant hydraulique - Alcali-activation Résistance à la compression - Durabilité

\section{Introduction}

Le projet de réaménagement de la baie du Mont-Saint-Michel prévoit la mise en déblais de près d'un million et demi de $\mathrm{m}^{3}$ de sédiments qu'il est nécessaire de valoriser. Si le traditionnel débouché de ces sédiments était l'amendement des terres agricoles, DOI: $10.5150 / \mathrm{cmcm} .2009 .038-1$ 
l'importance du volume ainsi constitué lors des travaux a nécessité la recherche de voies nouvelles pour leur devenir (LECLERC, 2006). Une des alternatives proposées est la stabilisation des sédiments par ajout de liant pouzzolanique (LP) et alcali activation (AA) pour une réutilisation en sous couches dans les ouvrages de terrassement faisant partie de ce projet que constituent les voies d'accès au site, les parkings et aires de stationnement aménagées pour l'accueil des touristes mais aussi pour les voies piétonnes. L'emploi de liants pouzzolaniques combinés ou non à une solution activatrice a fait l'objet d'une étude de faisabilité et d'essais de durabilité (OBANA, 2004). Les liants utilisés ont été proposés par l'entreprise ARD-Normandie (ARD, site web) spécialisée dans les traitements de sols de surface. Dans cette étude, un traitement de surface a été opéré sur les mélanges de sédiments stabilisés afin d'améliorer la résistance superficielle. Tout d'abord une description des différents constituants des mélanges est donnée avec leurs caractéristiques. Puis s'ensuit la mise en œuvre des éprouvettes d'essai : préparation, dosage, mélange, confection et conservation. Ainsi des séries d'éprouvettes ont été réalisées puis soumises à des essais de caractérisation et de comportement mécanique : résistance à la compression simple, tenue au gel-dégel et au mouillage-séchage. A partir des résultats obtenus, une analyse a permis de juger de l'efficacité de la solution activatrice et par ailleurs de l'apport du produit de traitement de surface ou de cure.

\section{Constituants des mélanges stabilisés}

\subsection{Le sédiment à stabiliser : la tangue}

La tangue est le nom donné aux sédiments de la baie du Mont-Saint-Michel. Ce sont des sédiments très riches en carbonates $\left(>50 \% \mathrm{CaCO}_{3}\right)$, (LEMEE, 2006). La tangue a été prélevée dans les différentes zones de déblais du projet. Elle est composée de particules fines, son diamètre maximal n'excède pas $100 \mu \mathrm{m}$ et le tableau 1 suivant en situe les fractions granulométriques.

Tableau 1. Références granulométriques de la tangue.

\begin{tabular}{lllll}
\hline Fraction argileuse $(\boldsymbol{\%}<\mathbf{2} \boldsymbol{\mu m})$ & Fraction silteuse $(\mathbf{2} \boldsymbol{\mu \mathbf { m } < \% <}<\mathbf{6 4} \boldsymbol{\mu m})$ & $\mathbf{d}_{\mathbf{1 0}}(\boldsymbol{\mu m})$ & $\mathbf{d}_{\mathbf{5 0}}(\boldsymbol{\mu m})$ & $\mathbf{d}_{\mathbf{9 0}}(\boldsymbol{\mu m})$ \\
\hline 22 & 78 & 0.5 & 5.4 & 26.2 \\
\hline
\end{tabular}

\subsection{Le liant pouzzolanique : la cendre volante LFC}

Les cendres volantes utilisées dans l'étude sont issues de la combustion de chaudières à Lit Fluidisé Circulant (LFC). Elles se distinguent des cendres obtenues par les procédés de combustion classique par leur structure en feuillets, leur finesse supérieure à $10000 \mathrm{~cm}^{2} / \mathrm{g}$, leur surface $\mathrm{BET}>10 \mathrm{~m}^{2} / \mathrm{g}$ et leur réactivité d'indice égal à 0.9 à 28 jours. 


\subsection{La solution activatrice}

Les différentes compositions réalisées sont activées par une solution de soude caustique de concentration 10 mols. La solution a été préparée à partir d'hydroxyde de sodium $(\mathrm{NaOH})$ en pastille, pure, à plus de $98 \%$, diluée dans de l'eau déminéralisée de $\mathrm{pH}=6.5$.

\subsection{Le produit de cure : le produit VerreSol ${ }^{\circledR}$}

Le produit VerreSol est un produit de cure destiné à augmenter la résistance à l'usure des sols traités et à réduire leur porosité. C'est un verre liquide de la famille des géopolymères.

\section{Elaboration du matériau traité}

\subsection{Définition des dosages}

Les dosages sont exprimés en $\%$ de matières sèches de tangue. Pour la concentration de la solution activatrice, elle a été définie comme étant le rapport de la somme des ions de $\mathrm{NaOH}$ en mols (équivalent à la somme des ions de la solution mère) sur la somme des volumes d'eau libre contenue dans le mélange. Les mélanges réalisés sont donnés au tableau 2 suivant.

Tableau 2. Mélanges et dosages réalisés.

\begin{tabular}{lllll}
\hline Mélange & Référence & $\begin{array}{l}\text { Dosage en liant } \\
(\%)\end{array}$ & $\begin{array}{l}\text { Dosage en } \\
\mathbf{N a O H}\end{array}$ & $\begin{array}{l}\text { Teneur en eau } \\
(\mathbf{\%})\end{array}$ \\
\hline Liant pouzzolanique & LP & $8^{*}$ & sans & 20 \\
Alcali Activation & AA & $8^{* *}$ & 4 mols & 20 \\
\hline
\end{tabular}

Nota : *, ** Liants composés d'un mélange de cendres volantes et de chaux, produit ARD ${ }^{\circledR}$

\subsection{Mise en œuvre des constituants}

Le protocole de malaxage consiste à mélanger la tangue et le liant (poudres), puis ajouter l'eau et la solution activatrice sur une durée de $2 \mathrm{mn}$. Puis des éprouvettes cylindriques $4 \times 8 \mathrm{~cm}$ (élancement 2), sont moulées et compactées à une énergie équivalente de l'essai Proctor Normal (OBANA, 2004).

\subsection{Conditions de maturation et traitement de surface (TS)}

Les éprouvettes sont démoulées et conservées par la suite à $20^{\circ} \mathrm{C}$. Le produit de cure à raison de $3 \mathrm{~g}$ par éprouvette est appliqué après $24 \mathrm{~h}$ par pulvérisation en 2 passes (rotation de l'éprouvette). Ces échantillons traités sont référencés TS. 


\section{Essais mécaniques et de durabilité des mélanges}

L'évaluation de la résistance mécanique et de la durabilité s'est faite à travers les essais en compression simple sur des éprouvettes témoins et sur des éprouvettes ayant subi des cycles de gel-dégel et mouillage-séchage à des temps de conservation de 7, 14, 28 jours. Ces essais ont été menés selon les normes en vigueur. A titre de comparaison, les résistances obtenues à la compression Rc à 28 jours sont consignées dans le tableau 3.

Tableau 3. Valeurs comparées de Rc à 28 jours, exprimées en MPa.

\begin{tabular}{cccc}
\hline Référence du mélange & Rc témoin & Rc gel-dégel & Rc mouillage séchage \\
\hline LP & 1.09 & 1.63 & Eprouvettes \\
LP + TS & 1.03 & 1.62 & endommagées \\
AA & 0.80 & 1.20 & 1.72 \\
AA + TS & 0.81 & 1.12 & 1.58 \\
\hline
\end{tabular}

\section{Conclusions}

Les valeurs de résistance en compression à 28 jours varient de 0.8 à $1.1 \mathrm{MPa}$ pour les deux types de mélanges réalisés ce qui vis-à-vis des exigences premières de la construction routière et de remblaiement sont suffisantes. Le mélange au liant pouzzolanique seul, fournit des valeurs très proches de 1.1 MPa. L'étude de la durabilité des mélanges a montré que la tangue traitée au liant pouzzolanique résiste bien aux cycles de gel-dégel alors que des dégradations sont observées lors des tests de mouillage séchage. La tangue stabilisée par alcali activation résiste mieux à ces cycles mouillageséchage. Pour ces éprouvettes, des gains en performances mécaniques sont aussi obtenus pendant les cycles de gel-dégel bien que celles-ci présentent quelques dommages. Un compromis pourrait être recherché en ajustant le pourcentage de liant dans l'application de l'alcali activation. L'action du produit de cure est négligeable pour la résistance à la compression mais retarde l'apparition des dommages résultant des cycles de gel-dégel. Rappelons que ce produit de cure n'est pas un liant de structure.

\section{Références bibliographiques et sitographiques}

$\mathrm{ARD}$ (site web). ARD, la référence des sols stabilisés naturels. http://www.ardnormandie.fr.

LECLERC M. (2006). Valorisation de la tangue, recherche de solutions et analyse technico-économique. Mémoire de stage ENTPE, DDE Manche, 98 p.

LEMEE F. (2006). Traitement par alcali activation de sédiments fins marins non contaminés et à faible teneur en eau - Mise au point d'un procédé de stabilisation. Thèse de doctorat, Université de Caen Basse-Normandie, $187 \mathrm{p}$.

OBANA M. (2004). Traitement des vases du Mont-Saint-Michel par liant pouzzolanique et alcali-activation. Mémoire de DESS Génie Littoral, Portuaire et des Estuaires, Université de Caen Basse Normandie, 47 p. 